\title{
Quand les firmes divisent le monde - Représentations du territoire-monde sur les sites internet des firmes transnationales
}

When the firms divide the world: Representations of the world territory on the websites of transnational firms

Wenn die Firmen die Welt unterteilen; Darstellung des Weltterritoriums auf den Internetseiten der transnationalen Firmen

\section{Clarisse Didelon}

\section{OpenEdition}

Journals

Édition électronique

URL : http://journals.openedition.org/rge/2957

DOI : $10.4000 /$ rge.2957

ISSN : 2108-6478

Éditeur

Association des géographes de l'Est

Édition imprimée

Date de publication : 31 octobre 2010

ISSN : 0035-3213

\section{Référence électronique}

Clarisse Didelon, «Quand les firmes divisent le monde - Représentations du territoire-monde sur les sites internet des firmes transnationales », Revue Géographique de l'Est [En ligne], vol. 50 / 1-2 | 2010, mis en ligne le 30 septembre 2011, consulté le 08 septembre 2020. URL : http://

journals.openedition.org/rge/2957 ; DOI : https://doi.org/10.4000/rge.2957

Ce document a été généré automatiquement le 8 septembre 2020

Tous droits réservés 


\title{
Quand les firmes divisent le monde - Représentations du territoire- monde sur les sites internet des firmes transnationales
}

\author{
When the firms divide the world: Representations of the world territory on the \\ websites of transnational firms
}

Wenn die Firmen die Welt unterteilen; Darstellung des Weltterritoriums auf den Internetseiten der transnationalen Firmen

Clarisse Didelon

\section{Introduction}

Du fait de leur poids économique, les entreprises transnationales font partie des principaux acteurs de la mondialisation. Elles détiennent également un pouvoir sur les territoires, notamment du fait de leurs choix en matière de localisation. Mais elles détiennent également un pouvoir politique et idéologique : comme le souligne, L. Carroué (2002), le déploiement de «leurs stratégies territoriales exacerbe (...) les rapports de domination entre les pôles de commandement, périphéries intégrées plus ou moins autonomes et espaces totalement marginalisés ». A l'échelle globale il existe une expression particulièrement intéressante $\mathrm{du}$ pouvoir des entreprises transnationales : afin d'accompagner leur stratégie de localisation et l'organisation de leurs activités, les transnationales produisent des découpages opérationels du monde. Ces découpages fonctionnels sont dictés par les stratégies des entreprises et par des lignes de partage incontournables tels que celles induites par les droits de douane, les codes des investissements, les legislations en vigueur, les formes d'intégration régionales, la taille et les dynamismes des marchés. Les entreprises s'organisent, par ailleurs, essentiellement en fonction des zones d'intégration régionales (Union 
Européenne, ALEAN, ASEAN etc.). Toutefois, les différents supports utilisés par les entreprises (rapports d'activités, plaquettes de présentation, sites internet, etc.) afin de présenter leurs activités, la répartition de leur masse salariale, ou plus simplement, d'indiquer les lieux où leurs filiales sont présentes produisent des découpages cartographiques - ou non (sous forme de listes et de tableaux) du monde. Ces cartes et tableaux sont le fruit de leurs services de communication ou de prestataires de services extérieurs. Toutefois nous pouvons supposer que ce matériel est validé au sein de l'entreprise avant toute diffusion. Dès lors, puisque diviser l'espace est une des modalités de l'exercice du pouvoir, les découpages cartographiques du monde peuvent rendre comptent du pouvoir, sinon pratique, du moins symbolique des entreprises transnationales comme acteurs territoriaux. A ce titre, de tels découpages contiennent une réelle dimension géopolitique. Nous nous proposons d'analyser ici les découpages du monde disponibles sur les sites Internet des entreprises transnationales qui faisaient partie des 500 plus grandes entreprises mondiales en 2009. L'analyse de ce matériel permet d'appréhender dans une certaine mesure le rapport que les entreprises entretiennent avec l'espace mondial. L'analyse de ces régionalisations du monde nous donne à voir la manière dont l'espace mondial est interprété, représenté et éventuellement pratiqué par les entreprises transnationales, lui donnant ainsi une dimension territoriale. Ce travail exploratoire cherche avant tout à déterminer quelles sont les représentations du monde rendues publiques par les firmes transnationales dans leurs documents accessibles au grand public: dans un première partie, nous expliciterons l'approche qui a guidé cette analyse avant de présenter la méthodologie suivie pour constituer notre base de données et pour l'analyser. Enfin, nous présenterons l'analyse des découpages du monde rendus publics sur les sites internet des entrerpises transnationales.

\section{Firmes transnationales, cartographie et pouvoir}

2 La mondialisation transforme le rapport des entreprises à l'espace géographique surtout par l'introduction de l'échelle mondiale dans leur représentation et leur action, " puisqu'elles doivent se projeter à des niveaux d'échelles inédits et vertigineux, ceux du vaste monde » (Bost, 2006). Le déploiement de leur action à l'échelle mondiale est souvent accompagné de la production de cartes de régions du monde. Découper le monde peut être considéré comme une expression du pouvoir, en ce que cette action manifeste à la fois un acte d'appropriation, mais aussi l'expression publique d'une certaine vision du monde.

\section{A. Un acte d'appropriation}

3 Le principal acteur du partitionnement d'un espace approprié est l'Etat qui découpe son territoire en entités administratives (régions, départements etc.) pour l'administrer et le contrôler. A l'échelle mondiale si l'Etat est considéré comme le premier niveau de mailles (Brunet, Ferras, \& Théry, 1993), les acteurs mondiaux divisent cet espace en régions de niveau supérieur à l'échelle étatique. Or à cette échelle, aucun acteur n'a le monopole d'une division du monde et de nombreuses régionalisations coexistent. Un premier type de divisions du monde est constitué des divisions conceptuelles, souvent produites à des fins cognitives ou explicatives par des 
acteurs mais surtout des observateurs politiques. Elles sont basées sur les métagéographies, c'est-à-dire sur « un ensemble de structures spatiales à travers lesquelles les personnes ordonnent leur connaissance du monde " (Lewis \& Wigen, 1997). Le partage du monde en un «Est " / et un « Ouest », hérité de la guerre froide fait partie des divisions conceptuelles, ainsi que la division « cœur, semi-périphérie, périphérie ». Le découpage du monde en civilisations, hérité de la logique classificatoire des lumières, développé par F. Braudel (1987) et R. Breton (1987) prend sous la plume de S. Huntington (2000) une dimension idéologique du fait de la perspective d'affrontement qui lui est corrélé. Un second type de divisions du monde est constitué des divisions fonctionnelles produites à des fins pratiques par des acteurs qui déploient une activité à cette échelle, ne serait-ce que la collecte de statistiques. Parmi celles-ci on trouve les divisions du monde en unités statistiques produites par l'ONU et ses nombreuses agences. Ces divisions peuvent sembler neutres mais elles induisent également la mobilisation de méta-géographies dans leur construction (Didelon, Grasland, \& Richard, 2008). C'est dans cette catégorie que nous trouvons les découpages produits par les entreprises.

4 L'action de représenter et surtout de diviser le monde en différentes parties n'est pas un acte neutre d'un point de vue géopolitique (Harley, 1995). En premier lieu la constitution d'unités spatiales, d'un réseau de mailles est une manifestation de l'appropriation d'un espace et de ses ressources, même lorsqu'il s'agit de l'espace mondial. En effet, «la maîtrise du territoire, de ses ressources tant humaines que physiques nécessite sa partition dès lors que l'on atteint une certaine masse et un certain degré de complexité » (Brunet, Ferras, \& Théry, 1993). Ainsi, le découpage permet de segmenter l'espace et les marchés des entreprises, en créant des régions de tailles plus maîtrisables, dans lesquelles l'entreprise va disposer de relais de pouvoir, les directions régionales. Cette réduction d'un espace de grande taille en entités plus petites est donc un moyen de rendre plus efficace son contrôle et sa gestion. Enfin, l'utilisation de ces divisions par les acteurs mondiaux contribuent à façonner le monde. En tant qu'outils opérationnels, les mailles crées vont peser sur l'action des entreprises et contribuer à homogénéiser chaque maille et à la différencier des autres.

\section{B. L'expression d'une vision du monde}

5 A l'échelle mondiale, comme aux échelles infranationales, les mailles tracées sont toujours le reflet de constructions spatiales enracinées dans les temps long de l'histoire (Lévy \& Lussault, 2003). Une division du monde est la projection d'une vision du monde qui classe les différent lieux ou espaces en fonction d'un point de vue spécifique. La production de découpages du monde implique une vision sous-jacente à laquelle on essaye de se conformer en postulant l'existence d'un ensemble géographique et en cherchant à le délimiter sur la foi de son nom (Lévy \& Lussault, 2003), comme lorsque l'on cherche à créer une région "Europe ». On peut également vouloir rassembler dans une même maille des espaces que l'on juge semblables selon certains critères. Dans ce cas, les représentations interviennent dans les critères choisis dans le processus de construction des zones. Ainsi, comme le souligne P. Poncet (2008), «les lignes de partage du monde contemporain sont une fiction cartographique " et elles ont deux fonctions : rassembler ce qui se ressemble ou séparer ce qui ne se ressemble pas. 
Or, ces cartes sont des objets publics, affichées sur les sites Internet des entreprises. Elles ont donc nécessairement un sens pour les acteurs qui les affichent, au moins en tant que signe ou symbole de leur pouvoir à l'échelle mondiale. Elles sont donc le reflet et l'expression d'une représentation du monde. Ainsi, le point de vue adopté par les entreprises transnationales est porteur d'une vision du monde, ne serait-ce que par le type de projection adopté, le planisphère imposant subrepticement une subjectivité et constituant en lui-même un message (Monmonnier, 1993).

\section{Source et construction des bases de données}

7 Les 222 entreprises dont nous avons analysé les représentations du monde, font partie des 500 plus grandes entreprises mondiales selon le magazine « Fortune $»^{1}$ en 2009. Les 500 plus grandes entreprises sont localisées dans 36 pays, principalement aux EtatsUnis, au Japon, en France et en Allemagne, et se répartissent dans 56 secteurs d'activités, les plus représentés étant la banque et l'assurance, le pétrole, l'automobile, l'agro-alimentaire et les télécommunications (voir tableau en annexe). Certaines d'entre elles (89) ne sont pas des entreprises transnationales et ne se déploient que sur un marché national. Afin de ne pas fausser les analyses nous avons pris la décision de les extraire de l'échantillon. Ainsi, nous avons considéré comme des entreprises transnationales, les entreprises qui étaient effectivement présentes dans plus d'un pays (Bost, 2006). Cela n'empêche pas, bien entendu, qu'elles aient un fort ancrage national (Carroué, 2002), mais le monde est pour elles un espace pertinent. Afin de construire notre base de données, le site internet de chacune des entreprises a été consulté afin d'y trouver une carte représentant le monde. Certaines entreprises (65) produisent un découpage du monde sous forme de liste ou de tableau. Compte tenu, de l'importance que nous avons choisi d'accorder à la représentation cartographique, nous ne les avons pas pris en compte dans l'analyse. La première carte trouvée sur le site Internet, et donc la carte la plus exposée au regard du public, a été copiée afin d'être analysée ultérieurement. Les cartes sont relativement faciles à trouver: elles sont souvent situées sur la page d'accueil, sinon, un onglet "présence mondiale » permet de les trouver facilement. Si la recherche sur le site Internet n'aboutissait pas, le rapport d'activité en ligne était également consulté : dans l'échantillon de 211 entreprises produisant une carte, 11 la propose uniquement dans leur rapport d'activité. Notre échantillon se compose donc de 222 cartes du monde receuillies sur les sites internet ou dans les rapports d'activités en ligne des entreprises transnationales².

$8 \quad$ A partir du relevé des cartes, deux bases de données ont été construites afin de mener notre analyse. La première permet d'analyser le fait que les entreprises produisent ou non une carte, le type de projection utilisé, le centrage, la création d'une région nationale ou non et enfin le nombre de régions formées. Associée à des renseignements sur l'entreprise (origine, secteur d'activité) cette base permet de conduire des tests sur l'existence de relations statistiques notamment de type test du Chi2. La seconde base de données est une matrice de coappartenance des pays du monde aux régions formées par les entreprises (Didelon \& Grasland, 2006). Pour chacune des 75 entreprises proposant une carte avec une régionalisation délimitée ${ }^{3}$ nous permettant donc de recueillir les données, une matrice de coappartenance des pays a été créée à partir du fichier de départ qui pour chaque entreprise reportait en face de chaque pays, le nom 
de la région dans laquelle il était inclus. Ensuite l'analyse de la régionalisation a été réalisée à l'aide d'une analyse en composante principales sur la matrice totale.

\section{Résultats et analyse}

\section{A. A quoi servent les cartes?}

Sur les 411 entreprises de l'échantillon une petite majorité d'entre elles, (222, soit $54 \% . .$.$) produit effectivement une carte. Les entreprises originaires de pays européens$ (Allemagne, France, Italie, Espagne, Grande-Bretagne) ont une tendance plus marquée à faire des cartes tandis qu'à l'inverse les cartes sont peu présentes sur les sites Internet des entreprises originaires d'Australie, du Canada, des Etats-Unis et de Russie. On peut $\mathrm{y}$ voir l'influence d'un héritage historique et culturel puisque les premiers sont des pays dont la tradition cartographique est ancienne. On ne note aucune relation particulière entre le secteur d'activité de l'entreprise et le fait qu'elle produise ou non des cartes.

La publication de cartes a le plus souvent une utilité pratique, bien que quelques rares cartes semblent avoir un but uniquement décoratif ou symbolique. Cette utilité est souligné par le fait que $57.6 \%$ d'entre elles sont interactives : cliquer sur une partie de la carte permet souvent dans un premier temps de "zoomer " sur un espace régional puis, dans un second temps, sur un pays et d'obtenir plus d'information sur ce lieu (part des employés ou du chiffre d'affaires, coordonnées des personnes à contacter). A part les cartes de « rien » $(7,66 \%)$ (figure $1 \& 2)$ dont l'objectif reste obscur trois grands types de cartes sont publiés sur les sites Internet : des cartes de présence, de localisation ou de régionalisation (figure $1 \& 2)$. Sur les cartes de localisation $(25,23 \%$, figure 2$)$ les entreprises marquent d'un point la présence de leurs bureaux ou de certaines de leurs activités (extraction pétrolière pour un pétrolier, port pour un armateur etc.). Ces cartes sont le fait d'entreprises qui déploient des activités dans l'espace mondial mais qui sont tributaires de flux (de matière première, de transport) et en particulier des entreprises de matières premières. Leur vision du monde est celle d'un espace unique composé des lieux fonctionnant en interaction. Un autre type de carte utilisé par près d'un quart des entreprises (figure 2) est la carte qui partage le monde en deux : d'un côté les pays où l'entreprise est présente (souvent représentés dans une belle couleur vive) et de l'autre ceux d'où elle absente (souvent dans un grisé un peu terne). Les entreprises des secteurs agroalimentaires ont une certaine propension à utiliser ce type de représentation. Mais la majorité des cartes (plus de 60\%, figure 2) sont des cartes proposant une régionalisation. Les entreprises du secteur automobile, de l'électronique et du commerce sont surreprésentées dans l'utilisation des cartes de régionalisation. Enfin, les cartes produites sur les sites Internet de certaines entreprises mixent les usages et peuvent combiner à la fois la localisation et la régionalisation ou la présence et la régionalisation grâce à une mise en œuvre assez aboutie de la sémiologie graphique. Cela explique pourquoi, la somme de la répartition des usages (figure 2) s'élève à plus de $100 \%$. 
Figure 1 : types de cartes

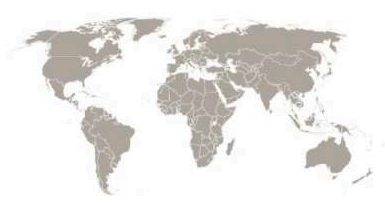

Carte « de rien »: Véolia

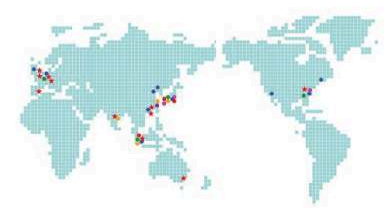

Carte de localisation : Mitsubishi Chemical
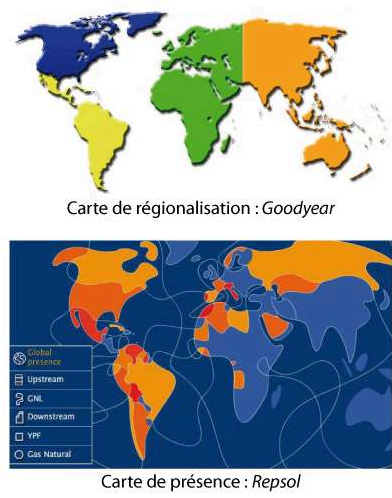

Figure 2 : les types de cartes produites par les entreprises

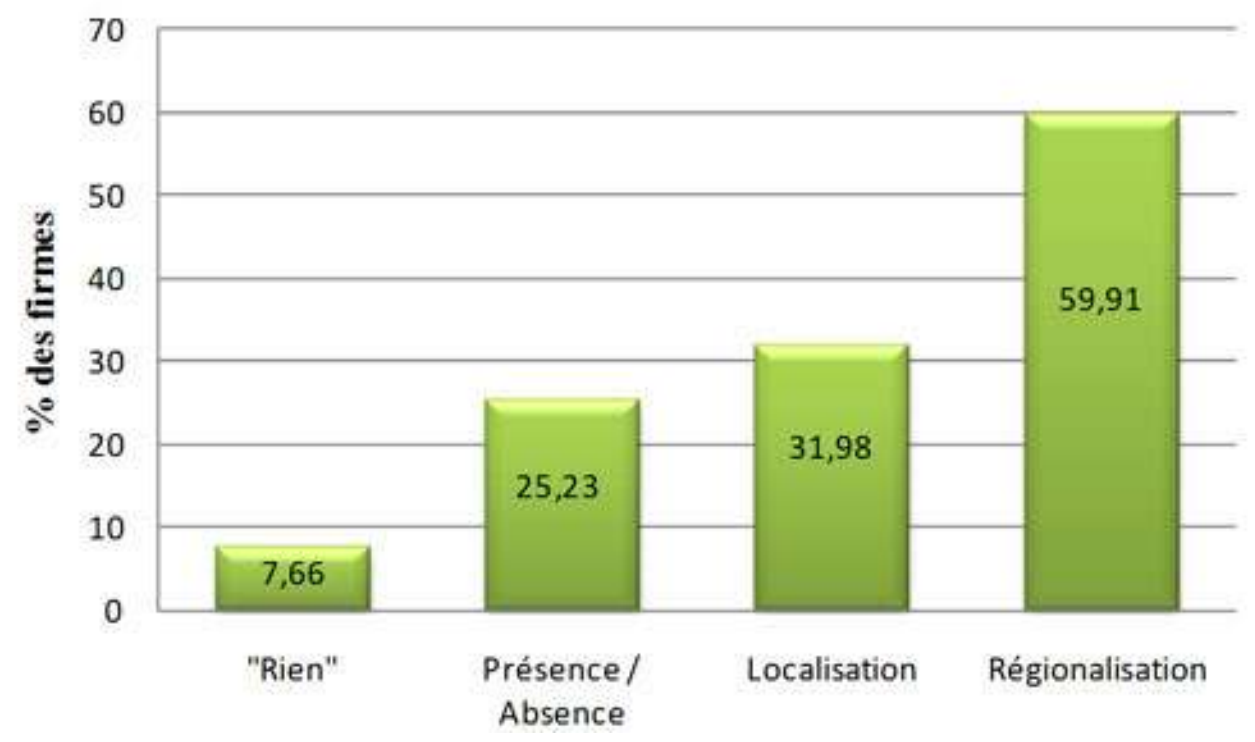

11 Le rôle utilitaire des cartes est souligné par l'analyse des liens hypertextes qui mènent aux cartes ou des titres de cartes quand elles se trouvent sur la page d'accueil ou dans un rapport d'activitét. La grande majorité des liens est composée du nom de l'entreprise suivi de worldwide ou in the world (Cisco worldwide, Air liquide in the world) ... Une analyse de fréquences montre que les trois termes les plus utilisés sont respectivement «worldwide» (46 fois sur 221), « global» ( 36 fois) et enfin « world» (29 fois). Si on y ajoute le terme un peu moins fréquent de «international» (14 fois), on remarque que les termes utilisés pour rendre compte du déploiement à l'échelle 
mondiale sont utilisés par près de $56 \%$ des entreprises. Trois autres termes sont fréquemment utilisés: "location", "presence " et "network». Ces observations nous conduisent à formuler l'hypothèse que, malgré l'alibi utilitaire, les cartes ont également un puissant rôle symbolique, celui de montrer le déploiement de l'entreprise à l'échelle mondiale.

\section{B. Représentation du monde}

Malgré les innovations que permet la présentation de cartes sur Internet, la plupart des cartes sont très classiques : plus de $97 \%$ d'entre elles sont " plates » contre moins de $3 \%$ globes animés (c'est-à-dire de globes représentés en 3D sur le site Internet et qui semblent tourner). Ces cartes sont aussi classiques dans leur représentation du monde, notamment dans la projection et la perspective utilisées. Quel que soit le pays d'origine, la majorité des entreprises utilise une projection de type Mercator. Cette projection, utilisée à foison dans les pays occidentaux, traduit la diffusion d'une représentation du monde conçue par les européens et souvent critiquée pour la place qu'elle accorde à l'Europe dans la représentation du Monde (Monastersky, 1992). Comme le souligne P. Poncet (2008), le fait de choisir une projection particulière parmi toutes les possibilités qui s'offrent au cartographe revient à «choisir un centre du Monde». La projection n'est donc pas neutre (Monmonnier, 1993) et le choix s'appuie "plus ou moins sciemment sur des a priori culturels ou idéologiques structurants » (Poncet, 2008).

\section{Centrage des cartes}

13 Cette prééminence du point de vue européen dans la représentation du monde est accentuée par le centrage utilisé. La grande majorité (75\%) des cartes sont centrées sur l'Europe (figure 3), ou du moins sur le méridien de Greenwich qui marque le triomphe du Royaume-Uni dans l'imposition de son système de coordonnées. Ainsi, quel que soit leur origine, la majorité des entreprises de notre échantillon produisent des cartes centrées sur l'Europe. Toutefois, le centrage semble être une question sensible pour un petit nombre d'entreprises: 5.73\% d'entre elles font le choix de ne pas centrer leur carte. Les solutions sont diverses (figure 3). La première, un peu expéditive traduit plus un centrage à l'extrême puisqu'il s'agit de produire une carte du monde tronquée, c'està-dire que certaines parties du monde ne sont tout simplement pas représentées. Onze entreprises, soit $4.4 \%$ ne figurent pas le monde en entier, mais seulement une partie (soit un centrage sur les bordures du Pacifique (New York life Insurance) ou de l'Atlantique (Wolseley), soit l'Eurasie (Auchan). Cette solution est rarement utilisée puisqu'elle trahit que l'extension mondiale de l'entreprise n'est pas achevée. La deuxième solution est de produire plusieurs cartes, trois (EADS) ou quatre (Alliance boots) par exemple, chacune incluse dans un cercle figurant le globe terrestre, et donnant des "vues» du monde. La multiplication de "vues", rend impossible la détermination d'un centre. La solution la plus élégante est bien entendu l'utilisation d'un globe animé où tous les lieux sont à peu près égaux dans la représentation bien que l'inclinaison du globe « cache " parfois certaines parties du monde, le plus souvent l'hémisphère sud. Enfin, une entreprise japonaise propose deux cartes du monde différentes selon la langue choisie sur la page d'accueil de son site Internet. Il s'agit de Hyundai Heavy Industries qui propose une carte centrée sur l'Asie sur la page d'accueil en Japonais et une carte centrée sur l'Europe sur la page d'accueil en Anglais. 
La prédominance de la vision euro centrique du monde est un phénomène relativement constant dans la cartographie du monde et ce quel que soit l'endroit où les cartes sont produites (Monastersky, 1992). Un peu plus de 14\% des entreprises produisent des cartes centrées sur l'Asie. Elles sont originaires de pays industrialisés asiatiques: la Corée du Sud, suivie de Taiwan et du Japon (figure 4). Les entreprises de ces pays produisent également des cartes centrées sur l'Europe, même si elles sont minoritaires (14\% pour la Corée du Sud, $33 \%$ pour Taiwan et $36 \%$ pour le Japon). Une seule entreprise d'un pays considéré comme occidental, le Canada, produit une carte centrée sur l'Asie. Il s'agit de d'une entreprise d'extraction minière (Husky Energy) dont le siège social se situe à Calgary et dont la majorité des activités d'extraction sont localisées dans l'ouest Canadien, ainsi qu'en Chine et en Indonésie. C'est donc, pour cette entreprise, une représentation plus commode qui minimise l'impression de dispersion de ses activités.

Figure 3 : type de centrage des cartes

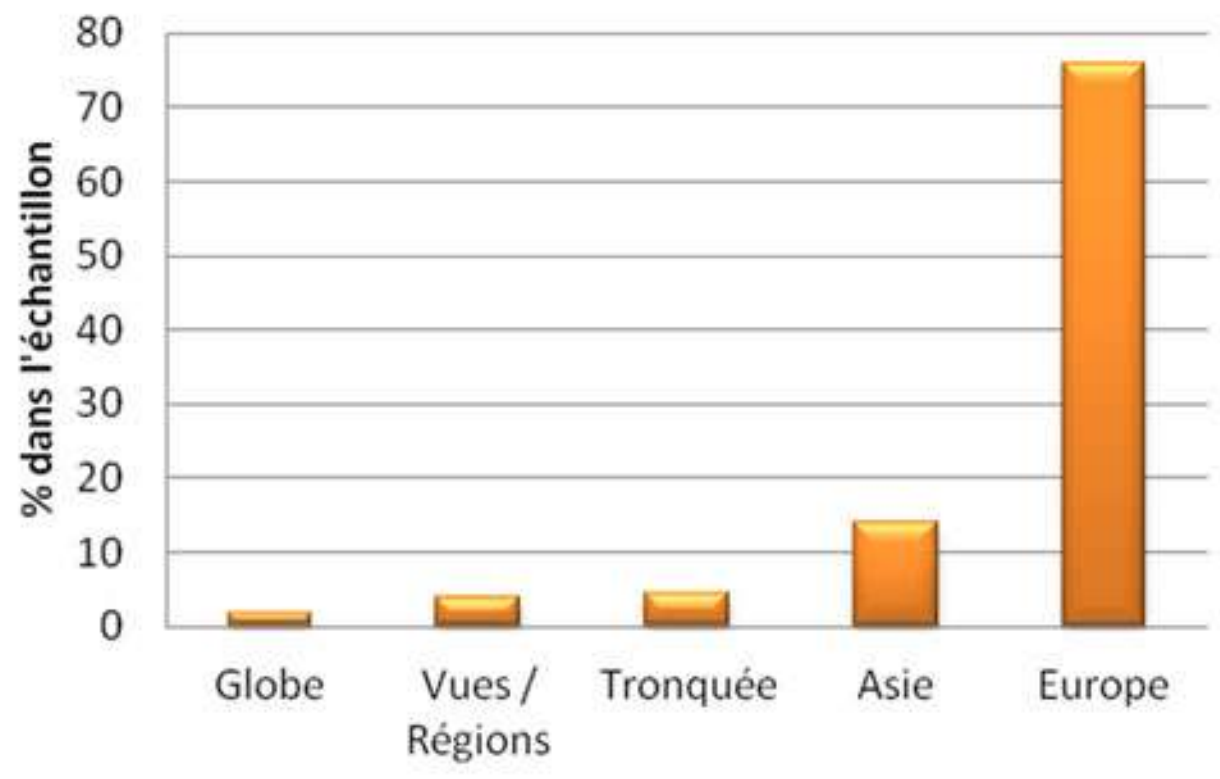


Figure 4 : origine des entreprises qui centrent leurs cartes sur l'Asie

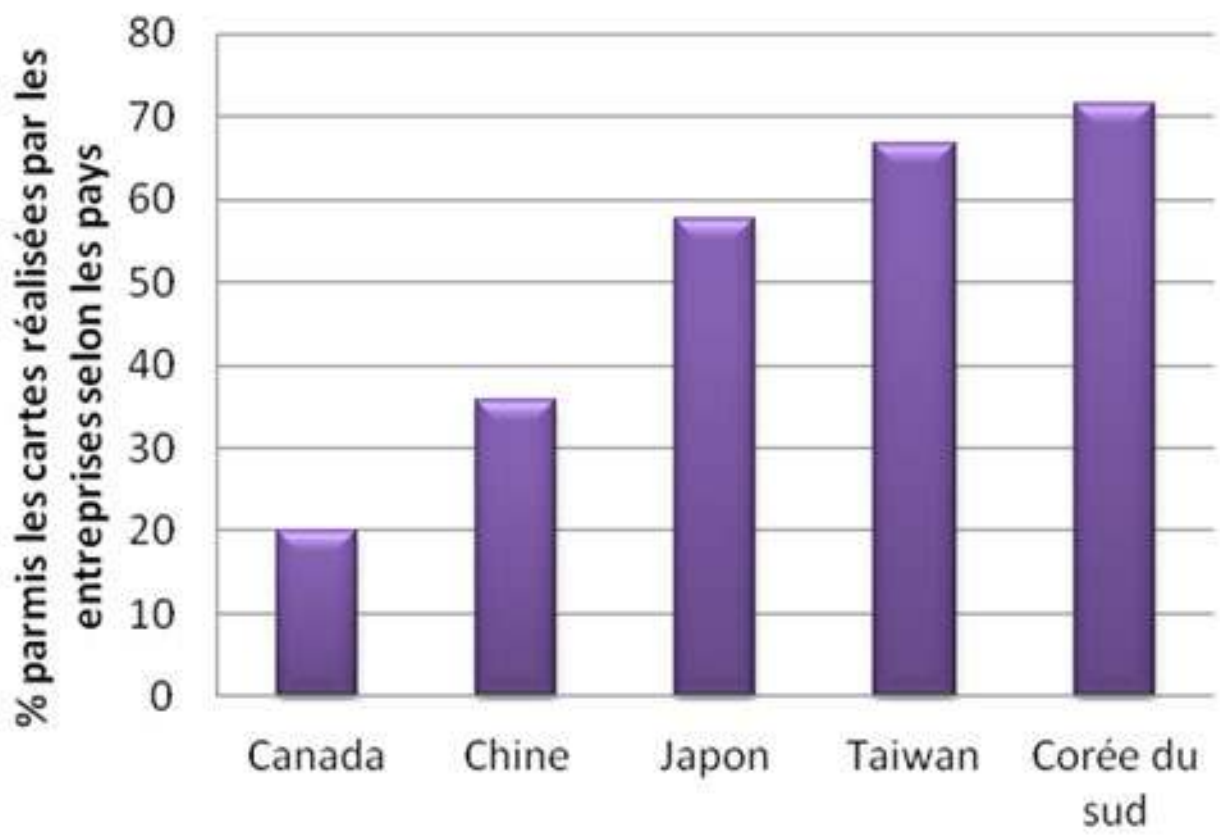

\section{Régionalisation}

Finalement quelles sont les régions du monde retenues par les entreprises dans leur représentation du monde? Les tracés des entreprises sont très variés, dans leur nombre comme dans leur extension. Les entreprises construisent entre 2 et 11 régions du monde, mais la majorité d'entre elles (près de 75\%) en font entre 4 et 6 (figure 5). Ces chiffres correspondent au nombre de continents traditionnellement enseignés : 5 continents dans la scolarité d'Europe, 6 ou 7 dans la scolarité d'Amérique du Nord (Grataloup, 2009). Toutefois toutes les régions dessinées ne correspondent pas à des figures continentales puisque certaines entreprises font une région qui inclut exclusivement leur pays. D'autres créent un certain nombre de régions uniquement là où elles sont plus ou moins présentes. Dans ce cas, une dernière zone est créée par défaut et parfois elle est nommée "reste du monde». C'est le cas pour l'entreprise Flextronic qui crée trois régions dans laquelle elle est présente (Amérique du Nord, Europe et Asie) et qui rassemble les autres pays dans le « reste du monde ». Par ailleurs, certaines entreprises créent des macro-régions pour lesquelles elles juxtaposent plusieurs noms (Afrique-Moyen Orient ou Amérique du Sud-Afrique). Il s'agit surtout des régions où elles sont peu présentes. Au final, le nombre de régions tracées, tout comme le nom qui leur est attribué, reflètent largement une vision continentale du monde qui est une vision héritée de l'héritage de la vision du monde européenne au moyen-âge (Grataloup, 2009). 
Figure 5 : nombre de régions identifiées par les entreprises

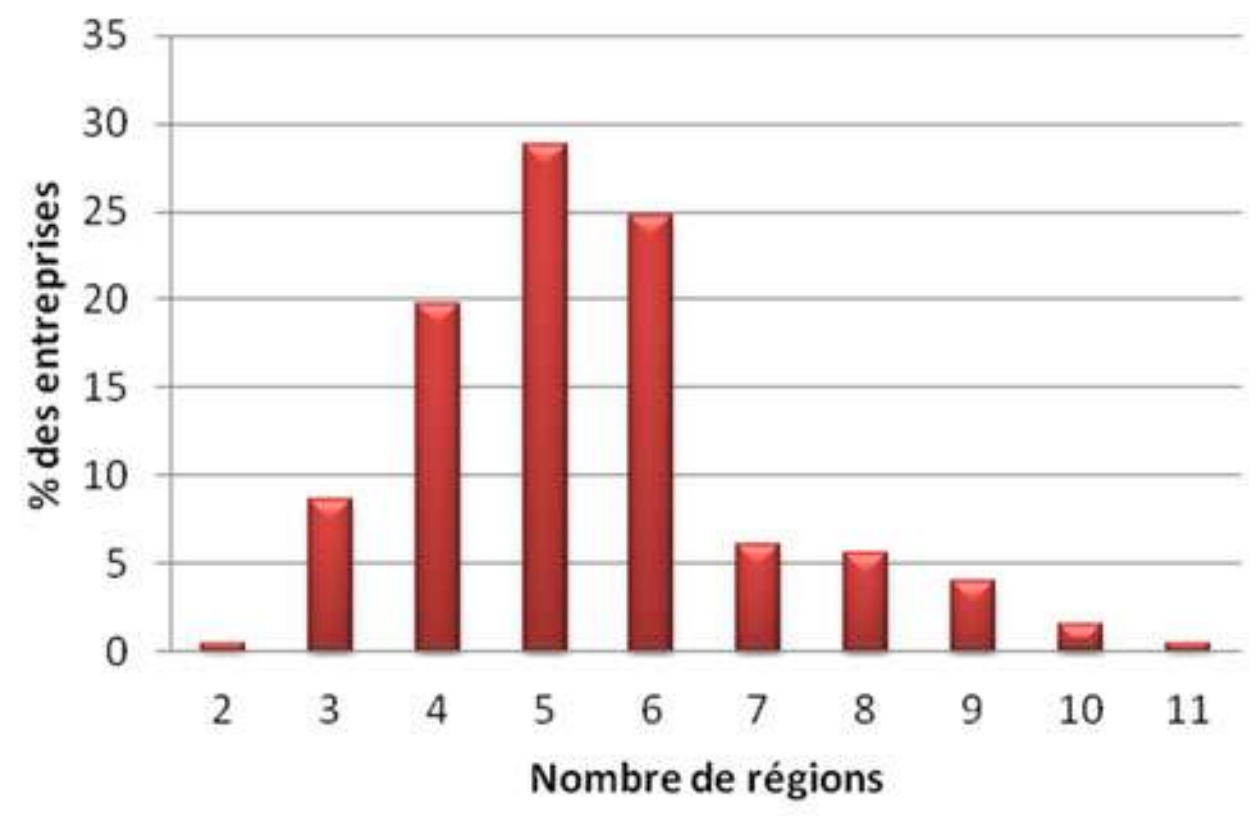

«Une région nationale »

Dans cet exercice de régionalisation du monde ${ }^{5}$, un aspect questionne de manière pertinente la relation que les entreprises entretiennent avec leur pays d'origine : la représentation de ce dernier comme région du monde. Cette question n'est pas anodine, puisque les entreprises transnationales sont soupçonnées tour à tour de ne suivre que leur propre intérêt ou alors de maintenir une coalition d'intérêts forte avec leur pays d'origine. Il ne faut pas oublier que "pour l'entreprise transnationale le contrôle de son territoire national demeure un impératif stratégique comme marché et base productive » (Carroué, 2002). Or, parmi les entreprises qui découpent le monde, un certain nombre isolent leur pays d'origine dans une seule et unique région. Elles sont 21 à le faire soit 10,6\% des entreprises, mais la répartition varie selon les pays d'origine des entreprises (figure 6) : ainsi, parmi les entreprises italiennes $33 \%$ font une région «Italie ", parmi les entreprises japonnaises $21,6 \%$ font une région «Japon », mais elles ne sont que 6,1\% des entreprises états-uniennes à faire une région «Etats-Unis ». Les entreprises délimitant une région nationale sont originaires de pays dont la « conscience nationale » est peut être considérée comme forte et qui revendiquent une place importante sur la scène internationale. Ce sont aussi des pays où le rôle du pouvoir politique est fort dans l'économie et dans le soutien aux grandes entreprises "fleurons" de l'industrie ou du commerce national. Toutefois, d'un point de vue statistique seules les entreprises japonnaises sont sur-représentées de manière significative ${ }^{6}$ dans la création d'une zone nationale. En effet, si la part du centrage sur l'Italie des entreprises italienne est la plus forte cela concerne un trop petit nombre d'entreprises pour être significatif. Pour le reste des entreprises, l'Etat d'origine semble donc avoir, d'un point de vue cartographique, relativement peu d'importance. Cela confirme, dans une certaine mesure le rôle symbolique que nous avons précédemment attribuée aux cartes celui d'affirmer la présence mondiale des entreprises. 
Figure 6 : part des cartes produisant une région « nationale » selon le pays

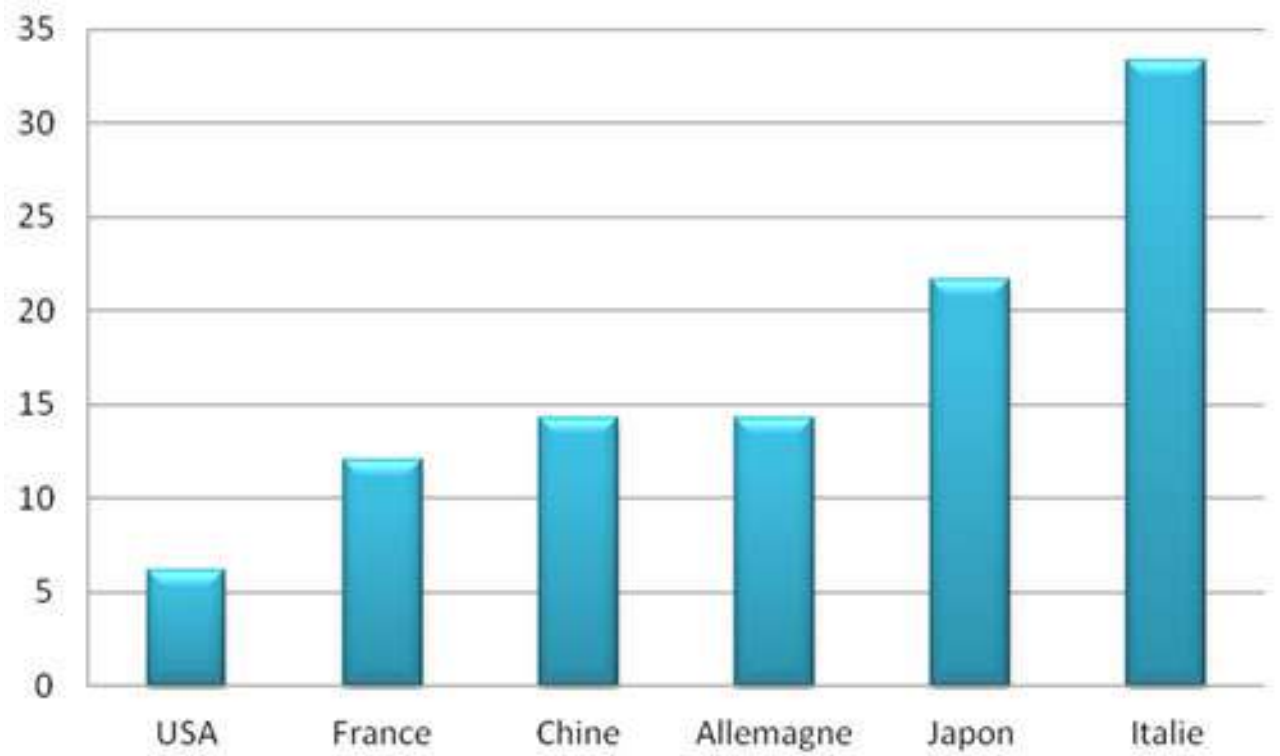

Quels sont les grandes zones d'ombre et d'interstices?

17 Pourtant, même en ayant un fort pouvoir symbolique, le monde est relativement maltraité par les entreprises transnationales. Cela s'exprime par le découpage de certains pays, mais aussi par l'oubli d'un grand nombre d'entre eux. La représentation cartographique des espaces oubliés et coupés est particulièrement intéressante d'un point de vue géopolitique (figure 7). Elle permet de reconstituer les régions qui présentent peu d'intérêt aux yeux des entreprises. Ainsi certaines régions qui constituent des marchés de première importance ne sont jamais oubliées (en vert). De grands marchés émergents stratégiques comme l'Inde sont très peu oubliés. D'autres, présentant relativement peu d'intérêt ou dans lesquels les obstacles culturels, économiques ou politiques sont importants sont plus souvent oubliées (comme l'Afrique centrale et l'Asie centrale).

Cela confirme que, en dépit de leur affichage mondial, les entreprises qui prennent réellement en compte l'ensemble de l'espace mondial sont finalement rares. Comme le souligne L. Carroué, (2002), "la logique de conquête de marché est extrêmement sélective : les transnationales valorisent prioritairement les marchés les plus puissants, les plus dynamiques et les plus stables. ». Cela se traduit par les oublis qu'elles commettent dans leur régionalisation du monde. 
Figure 7 : les espaces oubliés et coupés par les entreprises transnationales dans leur régionalisation du monde

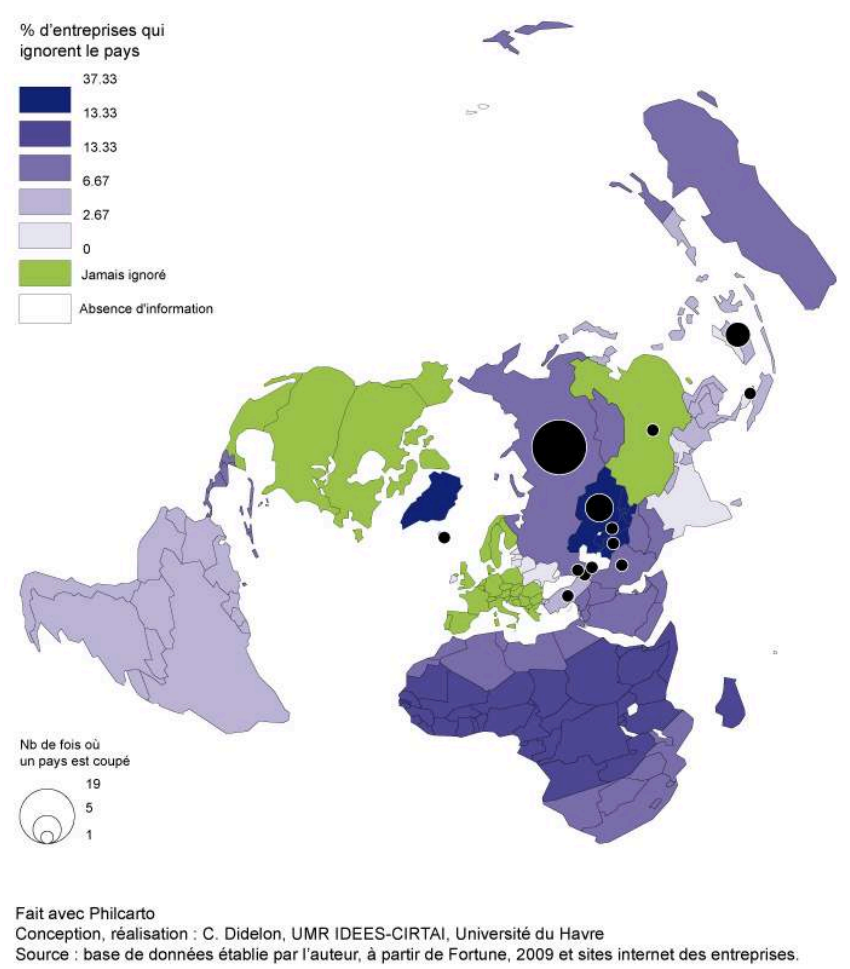

Un autre aspect est lié au découpage graphique de pays dans la régionalisation opérée. Bien entendu, ce sont les pays les plus étendus qui sont découpés et ceux localisés à l'intersection de deux régions géographiques. C'est également le cas de l'Indonésie, morcelée en une multitude d'îles, qui troublent le cartographe et peuvent le conduire à remettre en cause son intégrité territoriale en le divisant entre deux régions. Mais ce sont également des lieux de rivalités géopolitiques (notamment dans l'accès aux ressources) comme en Asie Centrale. La Russie est bien entendu coupée très souvent au niveau de l'Oural entre l'Europe et l'Asie. Ce découpage marque l'hésitation des cartographes quand à la région d'appartenance de certains pays notamment en ce qui concerne la Turquie (Europe? Asie ? Moyen Orient ?), mais il souligne également le peu de poids des pays ainsi «sacrifiés » sur la scène internationale. Il est à parier qu'aucune entreprise ne s'aviserait de couper en deux les Etats-Unis. En ce qui concerne les pays d'Asie centrale les réticences semblent être moins fortes.

\section{Quels sont les grands blocs?}

La partition du monde la plus significative d'un point de vue statistique est une partition en quatre parties du monde, qui rappelle la division continentale du monde encore en vigueur en France dans les programmes scolaires jusqu'aux années 1950 (Grataloup, 2009). Cette organisation par "continent» est le mode d'organisation dominant dans les entreprises transnationales qui partagent le «monde en grandes directions continentales (...) qui ont la responsabilité des activités industrielles et commerciales de leur périmètre géographique " (Carroué, 2002). Ainsi, les groupes continentaux sont privilégiés dans le dessin des régions, et certains constituent des blocs extrêmement robustes puisqu'ils resteront «entiers » jusqu'à la fin de l'analyse 
qui consiste à produire des classes de pays en fonction leur coappartenance régionale. Il s'agit des espaces à la forme relativement « ramassée » dont l'organisation interne est relativement mal connue et qui constituent pour les entreprises un marché relativement peu attractif. Il s'agit par exemple de l'Afrique. D'autres régions sont par contre l'objet de nombreuses subdivisions. C'est le cas de l'Asie qui apparait comme l'espace le moins robuste et le plus subdivisé. S'individualisent en premier un « Moyen Orient", une "Asie centrale», puis une "Océanie». Ensuite, à l'intersection de l'Europe et de l'Asie, la Turquie et les pays du Caucase forment une petite région intermédiaire. Un espace d'intersection similaire apparait également dans l'espace formé par l'Amérique centrale et les Caraïbes entre deux blocs plus robustes et donc plus souvent identifiés : l'Amérique du Nord et l'Amérique du Sud.

21 Ainsi, dans leurs découpages du monde, les entreprises privilégient les blocs continentaux, renforçant par là une vision européenne du monde, mais elles jonglent également avec quelques références culturelles que l'on peut déterminer grâce aux noms donnés (Moyen Orient, Amérique latine etc.) qui peuvent faire écho à une vision civilisationiste du monde. Certains espaces semblent plus « solides » face au découpage du monde, bien que ce soit aussi souvent les plus ignorés comme l'Afrique, d'autres sont plus «fragiles ", c'est-à-dire attribués à différentes régions selon les entreprises. Ce sont les espaces de "concurrence » entre deux grand blocs régionaux dont les marges sont pour le moins mouvantes dans les représentations cartographiques.

Figure 8 : grandes régions et zones intermédiaires dans la régionalisation du monde

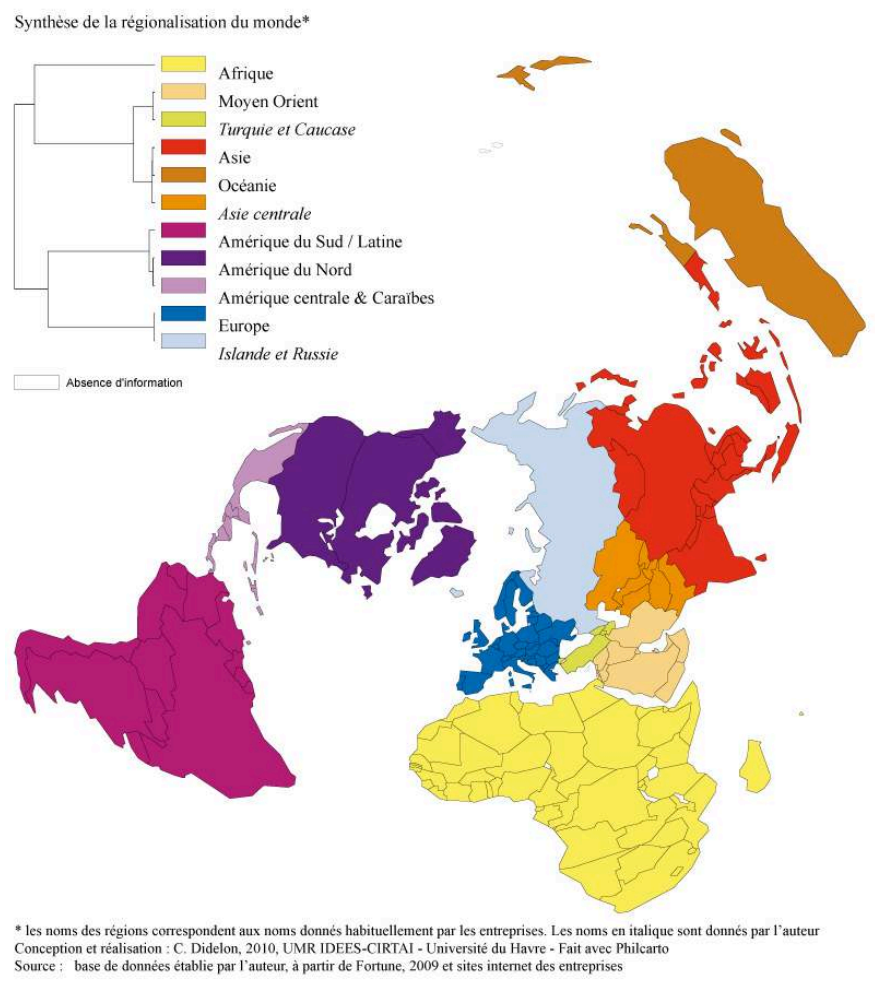

Revue Géographique de l'Est, vol. 50 / 1-2 | 2010 


\section{Conclusion}

L'analyse des cartes produites par les entreprises et de leurs découpages du monde montre que, quelle que soit leur origine, elles partagent majoritairement une vision continentale du monde, centrée sur l'Europe, et héritée de la vision classique que l'Europe a du monde. La prégnance de cet héritage historique est d'autant plus forte que les continents semblent «tout naturels » (Grataloup, 2009). Mais la diversité des points de vue sur le monde peut s'accroitre avec la mondialisation comme le montre la présence de quelques cartes centrées sur le Pacifique. Cette analyse est également intéressante dans la mesure où elle éclaire la relation entre les entreprises et l'espace mondial: la régionalisation du monde par les entreprises est à la fois une régionalisation cognitive puisqu'elle leur sert à comprendre le monde et à l'ordonner pour pouvoir le gérer, mais également le signe d'une subdivision performative d'un territoire (Poncet, 2008), c'est-à-dire que cette division peut servir de manière opérationnelle dans l'organisation et la gestion des activités de l'entreprise, contribuant ainsi à façonner l'espace mondial. Toutefois, les régionalisations présentées sur les sites I/internet des entreprises ne sont pas nécessairement les divisions opérationnelles utilisées par les entreprises, ce qui pose alors la question de la concordance entre la représentation et la pratique du territoire des entreprises à l'échelle mondiale. Ainsi, cette approche essentiellement descriptive et exploratoire, basée sur un matériel à la disposition du "grand public ", devrait bien entendu être complétée par une enquête approfondie au sein même des entreprises sur les stratégies qu'elles déploient dans la gestion de l'échelle mondiale.

Annexe : répartition des entreprises prises en compte dans l'analyse en fonction des secteurs d'activité

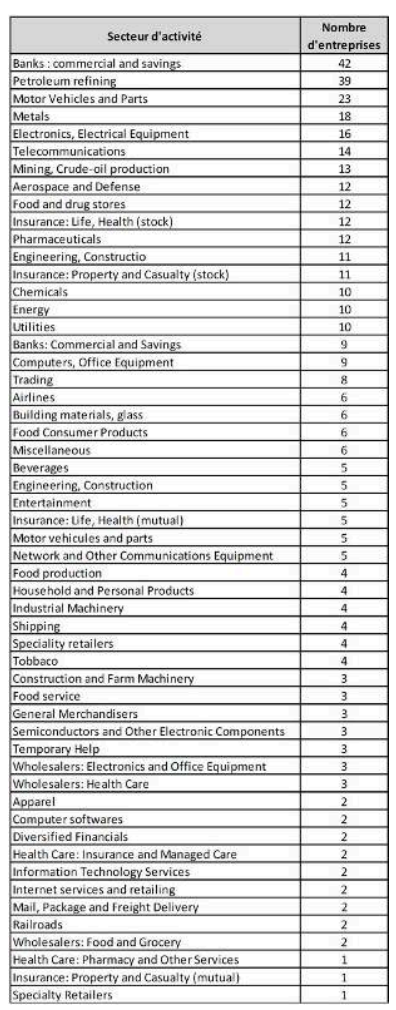




\section{BIBLIOGRAPHIE}

Bost F., 2006, « Entreprises, échelles et territoires face à mondialisation », in Carroué L., La mondialisation, Paris, SEDES, p.143-179.

Braudel F., 1987, Grammaire des civilisations, Paris, Arthaud

Breton R., 1987, Géographie des civilisations, Paris, Que sais je n² 2317, PUF, 127 p.

Brunet R., Ferras R., Théry H., 1993, Les mots de la géographie, Paris, La documentation française.

Carroué L., 2002, Géographie de la mondialisation, Paris, Armand Colin, Collection U.

Didelon C., Grasland C., 2006, Europe in the World, Final report, ESPON, Project 3.4.1. .

www.espon.eu.

Didelon C., Grasland C., Richard Y., 2008, Atlas de l'Europe dans le Monde, Paris, La Documentation française, Reclus.

Grataloup C., 2009, L'invention des continents, Paris, Larousse.

Harley B., 1995, « Cartes, savoir et pouvoir », in Gould P., Bailly A., Le pouvoir des cartes; Brian Harley et la cartographie, Paris, Anthopos.

Huntington S., 2000, Le choc des civilisations, Paris, Editions Odile Jacob.

Lévy J., Lussault M., 2003, Dictionnaire de géographie et de l'espace des sociétés,Paris, Belin.

Lewis M. W., Wigen K. E., 1997, The myth of continents, a critique od metageography, University of California Press.

Monastersky R., 1992, « The warped World of Mental Maps », Sceince News, 142 (14), p.222-223.

Monmonnier M., 1993, Comment faire mentir les cartes, ou du mauvais usage de la géographie, Paris, Flammarion.

Poncet P., 2008, « Partager le Monde », in Lévy J., L'invention du Monde, Paris, Les Presses de SciencePo, pp. 327-349.

Vilaça O., 2008, « La planète transactionnelle », in Lévy J., L'invention du Monde, Paris, Les Presses de SciencePo.

\section{NOTES}

1. http://money.cnn.com/magazines/fortune/

2. Compte tenu du choix explicite d'analyser les représentations cartographiques du monde rendues publiques sur les sites internet des entreprises cette posture ne produit en rien un biais dans l'échantillon.

3. Certaines entreprises produisent des listes de pays regroupés en régions. D’autres produisent des cartes de régions mais sans les limiter en plaçant uniquement un nom de région (Europe, Asie etc.) sur une carte du monde.

4. Pour mener cette analyse tous les textes ont été traduits en anglais

5. Pour cette analyse, 78 entreprises produisant une régionalisation cartographiée et dont les limites sont claires ont été utilisées. Parfois, pour éclaircir la situation d'un pays situé en limite 
de région, il a été nécessaire de cliquer sur ce pays pour vérifier à quelle région il avait été attribué.

6. Cette relation a été éprouvée par un test $\mathrm{du}^{\mathrm{Chi}}{ }^{2}$.

\section{RÉSUMÉS}

Les firmes transnationales produisent des cartes du monde qu'elles publient sur leurs sites Internet. Ces cartes, au-delà de leur aspect utilitaire, ont un rôle symbolique, celui d'affirmer leur présence mondiale et dans une certaine mesure leur pouvoir sur cet espace. Ce pouvoir s'exprime notamment par la réalisation d'une régionalisation qui diffuse une représentation du monde en même temps qu'il sert de cadre au déploiement des activités de l'entreprise contribuant dès lors à façonner l'espace mondial.

Transnational firms produce maps of the world to publish on their websites. These cards, in addition to their utilitarian aspect, have a symbolic role: they show their global presence and to some extent their power over this space. This power is expressed in particular through the implementation of regionalization, which broadcasts a representation of the world and it provides a framework for the deployment of business activities. Thus it helps to shape the global space.

Die transnationalen Firmen herstellen Weltkarten, die auf ihren Internetseiten veröffentlicht werden. Außerhalb ihrer Nutzung haben diese Karten eine symbolische Rolle: ihren Welteinfluss behaupten und in einem gewissen Ma $\beta$ ihre Macht über diesen Raum. Diese Macht kommt zum Ausdruck in der Herstellung einer Regionalisierung, die eine Darstellung der Welt verbreitet und die als Rahmen zur Entwicklung der Aktivitäten des Unternehmens benutzt wird, so dass sie zur Formung des Weltraumes beiträgt.

\section{INDEX}

Schlüsselwörter : Kartographie, Regionalisierung, transnationale Unternehmen, Welt

Mots-clés : cartographie, entreprises transnationales, monde, régionalisation

Keywords : mapping, regionalization, transnational corporations, world

\section{AUTEUR}

\section{CLARISSE DIDELON}

UMR IDEES - CIRTAI - Université du Havre - UFR LSH, 26 rue Philippe Lebon - 76600 Le Havre clarisse.didelon@univ-lehavre.fr 